BULLETIN OF THE

AMERICAN MATHEMATICAL SOCIETY

Volume 77, Number 4, July 1971

\title{
MANIFOLDS OF PIECEWISE LINEAR MAPS AND A RELATED NORMED LINEAR SPACE ${ }^{1}$
}

\author{
BY ROSS GEOGHEGAN ${ }^{2}$
}

Communicated by Michael F. Atiyah, December 21, 1970

1. Spaces of piecewise linear maps. Let $X$ and $Y$ be separable polyhedra, $X$ compact and $Y$ locally compact; for the moment let them be connected and of dimension $>0$. Denoting the separable hilbert space of square-summable sequences by $l_{2}$, a space is an $l_{2^{-}}$ manifold if separable, metrizable and locally homeomorphic to $l_{2}$. In [4] the author showed that the space $C(X, Y)$ of all continuous maps from $X$ to $Y$ with compact-open topology is an $l_{2}$-manifold. It is natural to ask whether the dense subspace $\operatorname{PL}(X, Y)$ consisting of all piecewise linear (p.l.) maps lies inside $C(X, Y)$ in some "nice" way. For example, is $\mathrm{PL}(X, Y)$ an infinite-dimensional manifold? and if so what is its model? and how are $\operatorname{PL}(X, Y)$ and $C(X, Y)$ related as manifolds?

To answer, let $l_{2}^{f}$ be the (dense, incomplete) linear subspace of $l_{2}$ consisting of those sequences having only finitely many nonzero entries. Then we claim that $\operatorname{PL}(X, Y)$ is an $l_{2}^{f}$-manifold. A pair $(M, N)$ is an $\left(l_{2}, l_{2}^{f}\right)$-manifold pair, if $M$ is an $l_{2}$-manifold for which there is an open cover $\mathcal{u}$ and open embeddings $\left\{f_{U}: U \rightarrow l_{2} \mid U \in \mathcal{u}\right\}$ such that for each $U \in \mathcal{U}, f_{U}(U \cap N)=f_{U}(U) \cap l_{2}^{s}$. We claim that the pair $(C(X, Y), \mathrm{PL}(X, Y))$ is an $\left(l_{2}, l_{2}^{f}\right)$-manifold pair. Among other things, it follows that $\operatorname{PL}(X, Y)$ has a (metric) triangulation, and that if $\operatorname{PL}(X, Y)$ is contractible, then it is homeomorphic to $l_{2}^{f}$.

2. Application : a normed linear space. Before giving more details, we give a simple application. Consider $\operatorname{PL}(I, R)$, the space of p.l. paths in the real line, with the usual vector space structure. We have claimed that $\operatorname{PL}(I, R)$ is homeomorphic to $l_{2}^{f}$; but whereas the linear dimension of $l_{2}^{f}$ is $\boldsymbol{\aleph}_{0}$, the linear dimension of $\operatorname{PL}(I, R)$ is $c$. (Proof:

AMS 1970 subject classifications. Primary 57A20, 58D15; Secondary 57C99, 54C35, 58B05, 46B05, 46E10.

Key words and phrases. Metric simplicial complex, triangulated infinite-dimensional manifold, function space, piecewise linear map, manifold of maps, sigmacompact linear space.

1 Research partially supported by NSF Grant GP 7952 X2.

${ }^{2}$ A special case of the theorem announced here is in the author's Ph.D. thesis written under Professor David W. Henderson at Cornell University. Supported by an I.B.M. Graduate Fellowship. 
suppose $B$ is a linear basis of cardinality $\boldsymbol{\aleph}<c$; then for each $g \in B$, let $A_{g}$ be the finite set of vertices of the minimal subdivision of $I$ on which $g$ is linear; $\cup\left\{A_{g} \mid g \in B\right\}$ has cardinality $\boldsymbol{N}$, so there exists $x \in I$ which lies in no $A_{g}$; any p.l. map which is not linear on a neighborhood of $x$ cannot be a finite linear combination of elements of $B$; this contradicts the fact that $B$ is a linear basis.) Summarizing we have

EXAMPLE. The normed linear space $\operatorname{PL}(I, R)$ is homeomorphic to $l_{2}^{f}$ but has a different linear dimension.

We mention this Example because we are told it is the first of its kind. Until recently it had been conjectured that any $\sigma$-compact normed linear space is topologically and linearly equivalent either to $l_{2}^{f}$ or to the subspace of $l_{2}$ consisting of sequences $\left(x_{1}, x_{2}, \cdots\right)$ such that $0 \leqq x_{i} \leqq 1 / 2^{i}$ for all but a finite number of entries $x_{i}$. Spurred on by the above counterexample, Henderson and Pełczyński [5] have discovered an uncountable collection of $\sigma$-compact prehilbert spaces, no two of which are homeomorphic.

3. The background. By a topological characterization of $l_{2}^{f}$ in $l_{2}$ we mean a set of topological conditions such that if $M$ is homeomorphic to $l_{2}$ while $N \subset M$ satisfies the conditions, then $(M, N)$ is homeomorphic (as a pair) to $\left(l_{2}, l_{2}^{f}\right)$. Independently, Anderson in [1] and Bessaga-Pełczyński in [2] gave such a topological characterization. The work of Torunczyk in [7] and [8] is closely related. Developing their ideas, West gave the following topological characterization of $\left(l_{2}, l_{2}\right)$-manifold pairs :

Theorem 1 (SeE Theorem 6 of [9]). Let $(M, d)$ be a metric space and let $N \subset M$. The pair $(M, N)$ is an $\left(l_{2}, l_{2}^{f}\right)$-manifold pair if and only if

(i) $M$ is an $l_{2}$-manifold,

(ii) $N$ is the countable union of finite-dimensional compacta,

(iii) given $\epsilon>0$, a pair $(A, B)$ of finite-dimensional compacta and an embedding $e: A \rightarrow M$ such that $e(B) \subset N$, there exists an embedding $e^{\prime}: A \rightarrow M$ such that $e^{\prime}(A) \subset N, e^{\prime}|B=e| B$ and $d\left(e(x), e^{\prime}(x)\right)<\epsilon$ for all $x \in A$.

We should mention here that $l_{2}^{f}$-manifolds and $\left(l_{2}, l_{2}^{f}\right)$-manifold pairs have been studied by Henderson-West (an extensive list of properties appears in [6]) and by Chapman [3].

4. The Main Theorem. Throughout, we use the notation $C\left(\left(X, X^{\prime}\right),\left(Y, Y^{\prime}\right)\right)$ for the space of continuous functions from the pair $\left(X, X^{\prime}\right)$ to the pair $\left(Y, Y^{\prime}\right)$, but if $X^{\prime}$ is empty we abbreviate to $C(X, Y)$. In the case of polyhedral pairs, we denote the corresponding spaces of p.l. maps by $\operatorname{PL}\left(\left(X, X^{\prime}\right),\left(Y, Y^{\prime}\right)\right)$ and $\operatorname{PL}(X, Y)$. 
Before stating the Main Theorem we recall the following special case of Theorem (4.7) of [4]:

Theorem 2. Let $K$ and $L$ be simplicial complexes with $K$ finite and $L$ locally finite; let $|K|$ have dimension $>0$, let each component of $|L|$ have dimension $>0$, let $K_{0}$ be a subcomplex of $K$, and let $p \in|L|$. Then $C\left(\left(|K|,\left|K_{0}\right|\right),(|L|,\{p\})\right)$ is an $l_{2}$-manifold. In particular, when $K_{0}$ is empty, $C(|K|,|L|)$ is an $l_{2}$-manifold.

In general, we do not know whether Theorem 2 holds when $\{p\}$ is replaced by a more general subpolyhedron. But there is one wellknown case given by Eells in [10] where it does hold. As in Theorem 2 , we only state the polyhedral case:

Theorem 3 (Eells). Let $K$ and $K_{0}$ be as in Theorem 2; let L triangulate a $C^{2}$-manifold of dimension $>0$; let $L_{0}$ be a subcomplex which triangulates a $C^{2}$-submanifold of $|L|$. Then $C\left(\left(|K|,\left|K_{0}\right|\right),\left(|L|,\left|L_{0}\right|\right)\right)$ is an $l_{2}$-manifold.

(Note. This way of stating Theorem 3 is valid because all separable Banach spaces are homeomorphic to $l_{2}$.)

The Main Theorem. Let $\left(K, K_{0}\right)$ and $\left(L, L_{0}\right)$ be pairs of simplicial complexes, where $K$ is finite and $L$ is locally finite. Let

$$
\begin{aligned}
& M=C\left(\left(|K|,\left|K_{0}\right|\right),\left(|L|,\left|L_{0}\right|\right)\right) \text { and } \\
& N=\operatorname{PL}\left(\left(|K|,\left|K_{0}\right|\right),\left(|L|,\left|L_{0}\right|\right)\right) .
\end{aligned}
$$

Then $(M, N)$ is an $\left(l_{2}, l_{2}^{f}\right)$-manifold pair whenever $M$ is an $l_{2}$-manifold.

OUTLINE OF THE PROOF. We remark that in view of Theorems 2 and $3, M$ is nearly always an $l_{2}$-manifold, so the theorem is widely applicable. We must check that the conditions of Theorem 1 hold for the pair $(M, N)$. Condition (i) holds by hypothesis. Condition (ii) is easily reduced to proving that, for each $k>0, \operatorname{PL}\left(\Delta^{k}, R\right)$ is the countable union of finite-dimensional compacta, where $\Delta^{k}$ is a euclidean $k$ simplex. This is not hard when $k=1$; and for any $k>0$ it is not hard to prove $\sigma$-compactness. The difficulty for $k>1$, and it is substantial, arises from the "finite-dimensional" requirement. We omit further details here. For condition (iii), the finite-dimensionality of $A$ and the fact that $M$ is an ANR are used to extend the embedding $e$ to a map $f: D \rightarrow M$ where $D$ is a compact polyhedron. Let $B^{\prime}=f^{-1}(B)$. By simplicial approximation of a special sort, replace $f$ by a nearby map $g: D \rightarrow M$ such that $g\left|B^{\prime}=f\right| B^{\prime}$ and $g\left(D-B^{\prime}\right) \cap g\left(B^{\prime}\right)=\varnothing$. It is also required of $g$ that for each $x \in D-B^{\prime}, g(x)$ is a p.l. map in $N$ which is 
nonconstant on some component of $|K|$ and is linear on the simplexes of a barycentric subdivision of $|K|$ (the mesh of the subdivision depending on the distance of $x$ from $B^{\prime}$ ). Then for each $x \in D-B^{\prime}$, a continuously varying p.l. homeomorphism $\psi(x)$ of $\left(|K|,\left|K_{0}\right|\right)$ is defined which is not the identity on any principal simplex of the particular barycentric subdivision of $|K|$ associated with the point $x$. This last condition ensures that $h(x)=g(x) \circ \psi(x)$ is different from $g(x)$. In fact, in this way, a map $h: D \rightarrow M$ is constructed so that $h$ is near $g, h\left|B^{\prime}=g\right| B^{\prime}=f \mid B^{\prime}, h\left(D-B^{\prime}\right) \cap h\left(B^{\prime}\right)=\varnothing, h\left(D-B^{\prime}\right)$ $C N$ and $h \mid\left(D-B^{\prime}\right)$ is injective. Once such a map $h$ has been constructed, it is clear that $e^{\prime}=h \mid A$ is the required embedding.

\section{REFERENCES}

1. R. D. Anderson, On sigma-compact subsets of infinite-dimensional spaces, Trans. Amer. Math. Soc. (to appear).

2. C. Bessaga and A. Pełczyński, Estimated extension theorem, homogeneous collections and skeletons, and their applications to topological classification of linear metric spaces, Fund. Math. 69 (1970), 153-190.

3. T. A. Chapman, Dense sigma-compact subsets of infinite-dimensional manifolds, Trans. Amer. Math. Soc. 154 (1971), 399-426.

4. R. Geoghegan, On spaces of homeomorphisms, embeddings and functions. I (to appear).

5. D. W. Henderson and A. Pełczyński, (to appear).

6. D. W. Henderson and J. E. West, Triangulated infinite-dimensional manifolds, Bull. Amer. Math. Soc. 76 (1970), 655-660.

7. H. Torunczyk, On estimated extensions of homeomorphisms in metric spaces, Bull. Acad. Polon. Sci. 18 (1970), 13-16.

8. - Skeletonized sets in complete metric spaces and homeomorphisms of the Hilbert cube, Bull. Acad. Polon. Sci. 18 (1970), 119-126.

9. J. E. West, The ambient homeomorphy of an incomplete subspace of infinitedimensional Hilbert spaces, Pacific J. Math. 34 (1970), 257-268.

10. J. Eells, Jr., On the geometry of function spaces, Internat. Sympos. on Algebraic Topology, Universidad Nacional Autónoma de México and UNESCO, Mexico City, 1958, pp. 303-308. MR 20 \#4878.

Institute for Advanced Study, Princeton, New Jersey 08540 